\title{
Metallic single-electron transistor without traditional tunnel barriers
}

\author{
V.A. Krupenin ${ }^{1}$, A.B. Zorin ${ }^{2,3}$, D.E. Presnov ${ }^{2}$, M.N. Savvateev ${ }^{1}$, and J. Niemeyer ${ }^{3}$ \\ ${ }^{1}$ Laboratory of Cryoelectronics, Moscow State University, 119899 Moscow, Russia \\ ${ }^{2}$ Nuclear Physics Institute, Moscow State University, 119899 Moscow, Russia \\ ${ }^{3}$ Physikalisch-Technische Bundesanstalt, 38116 Braunschweig, Germany
}

\begin{abstract}
We report on a new type of single-electron transistor (SET) comprising two highly resistive $\mathrm{Cr}$ thin-film strips $(\sim 1 \mu \mathrm{m}$ long) connecting a $1 \mu \mathrm{m}$-long Al island to two Al outer electrodes. These resistors replace small-area oxide tunnel junctions of traditional SETs. Our transistor with a total asymptotic resistance of $110 \mathrm{k} \Omega$ showed a very sharp Coulomb blockade and reproducible, deep and strictly e-periodic gate modulation in wide ranges of bias currents $I$ and gate voltages $V_{g}$. In the Coulomb blockade region $(|V| \leq$ about $0.5 \mathrm{mV})$, we observed a strong suppression of the cotunneling current allowing appreciable modulation curves $V\left(V_{g}\right)$ to be measured at currents $I$ as low as $100 \mathrm{fA}$. The noise figure of our SET was found to be similar to that of typical $\mathrm{Al} / \mathrm{AlO}_{x} / \mathrm{Al}$ single-electron transistors, viz. $\delta Q \approx 5 \times 10^{-4} e / \sqrt{\mathrm{Hz}}$ at $10 \mathrm{~Hz}$.
\end{abstract}

\section{INTRODUCTION}

The single-electron transistor (SET) is a system of two ultra-small metal-insulator-metal tunnel junctions attached to a small island which is capacitively coupled to a gate electrode. Due to their considerable resistance, $R \gg R_{Q} \equiv h / 4 e^{2} \cong 6.5 \mathrm{k} \Omega$, the tunnel junctions ensure charge quantization on the island. On the other hand, the junctions still enable the (correlated) charging and discharging of the island by individual electrons when the temperature is sufficiently low, $k_{B} T \ll E_{c}$. Here $E_{c}=$ $e^{2} / 2 C_{\Sigma}$ is the charging energy and $C_{\Sigma}=C_{1}+C_{2}+C_{0}+C_{g}$ the total capacitance of the island which includes the capacitances of the junctions $C_{1,2}$, the self-capacitance of the island $C_{0}$ and the capacitance between the island and gate electrode $C_{g}$. Transport of electrons is controlled by the transistor gate polarizing the island and therefore changing the Coulomb blockade threshold. Increase in the gate voltage $V_{g}$ causes a stepping increment of the number of electrons on the island and this leads to eperiodic dependence of the $I-V$ characteristic on $V_{g}$. Due to this effect, the transistor provides a means for measuring the polarization charge on its island with subelectron accuracy. This property of SET was successfully exploited in many experiments to measure and monitor sub-electron quantities of charge in mesoscopic systems (see some examples in Refs. [1, 2, 3, 4, 5]). Different materials and methods have been used for the fabrication of SETs. Although substantial progress has been achieved in the fabrication techniques, there is still a demand for devices which have good electric parameters, are easy to fabricate and less subject to electrical shock destruction. In this paper we present a new type of SET electrometer having a potential to meet these requirements. The idea of our work was to fabricate and characterize a metallic transistor with high-ohmic $\left(R \gg R_{Q}\right)$ Cr-film microstrips replacing the traditional (oxide) tunnel barriers between island and outer electrodes. The junctions of this type should, in our opinion, also ensure sufficient isolation of the island, leading to correlated electron motion across the device [6]. Below we report on the single electron characteristics of our device.

\section{SAMPLE FABRICATION}

The structures were fabricated by shadow evaporation through a suspended mask [7] on Si substrate buffered by an $\mathrm{AlO}_{x}$ layer (about $200 \mathrm{~nm}$ thick). Since we aimed to obtain reliable metallic contacts between $\mathrm{Cr}$ and $\mathrm{Al}$ films, the fabrication process was carried out in situ in three steps. First, the Al film $10 \mathrm{~nm}$ thick was deposited by e-gun at the first angle. It formed the bottom layers of the island and outer electrodes. Secondly, the $\mathrm{Cr}$ film $6 \div 8 \mathrm{~nm}$ in thickness was evaporated at a small residual oxygen pressure $\left(\approx 10^{-5}\right.$ mbar $)$. This evaporation was performed at another angle to overlap the bottom $\mathrm{Al}$ layer and give rise to the electrical connection between the island and outer electrodes. The nominal contact area between the $\mathrm{Al}$ and $\mathrm{Cr}$ layers was $100 \mathrm{~nm}$ by $120 \mathrm{~nm}$. Finally, the second Al layer (about $30 \mathrm{~nm}$ thick) was evaporated at the first angle so that the ends of the $\mathrm{Cr}$ strips were clasped by $\mathrm{Al}$ electrodes from the bottom and from the top. The gate electrode, completing the transistor structure, obtained by evaporation of both $\mathrm{Al}$ and $\mathrm{Cr}$ films, and it was positioned near the island. The top view of the resulting structure is presented as a sketch in Fig. 1 where the stray metal shadows are not shown for the sake of clarity.

\section{CHARACTERIZATION OF CHROMIUM STRIPS}

Before studying the transistor structures we had characterized the detached $\mathrm{Cr}$ strips $(1.0 \div 1.4 \mu \mathrm{m}$ long, $100 \mathrm{~nm}$ wide and $6 \div 8 \mathrm{~nm}$ thick) identical to those used in the transistor samples. In order to check a possible influence of the electric field on the electron transport in $\mathrm{Cr}$ strips, each strip was supplied by an individual 


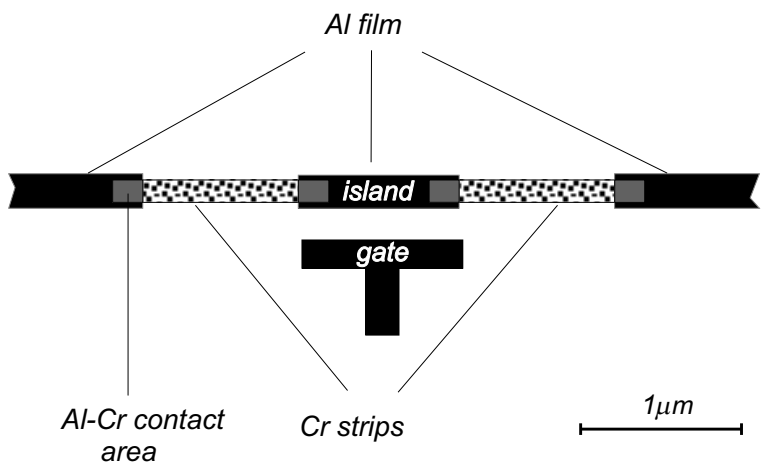

FIG. 1: The geometry (top view) of the Cr-film transistor structure. For clarity, the width of the strips (about $100 \mathrm{~nm}$ ) is shown somewhat larger.

gate electrode placed nearby. The $\mathrm{Cr}$ strips had different resistances per square $R_{\square}$ (from $2.5 \mathrm{k} \Omega$ to $14 \mathrm{k} \Omega$ ) depending on their thickness. The $I-V$ curves of these strips measured at $T=30 \mathrm{mK}$ are presented in Fig. 2 .

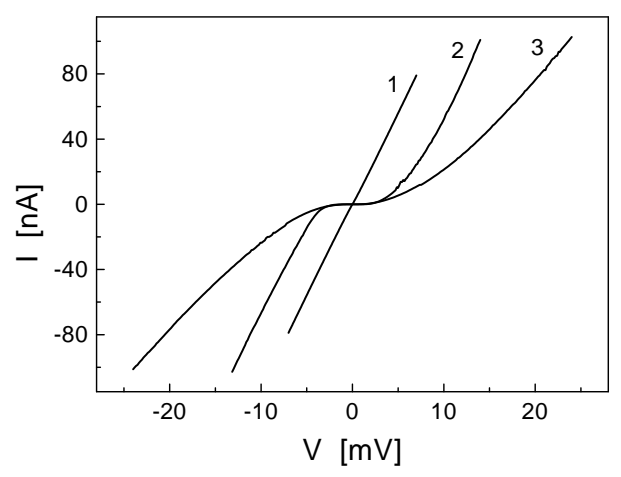

FIG. 2 : $I-V$ curves of $6 \div 8 \mathrm{~nm}$ thick Cr strips having different resistances per square $R_{\square}$. Curve 1: $1 \mu \mathrm{m}$-long low resistance strip, $R=25 \mathrm{k} \Omega\left(R_{\square} \approx 2.5 \mathrm{k} \Omega\right), V_{\text {off }} \approx 70 \mu \mathrm{V}$; Curve 2: $1.4 \mu \mathrm{m}$-long medium resistance strip, $R=80 \mathrm{k} \Omega\left(R_{\square} \approx\right.$ $5.5 \mathrm{k} \Omega), V_{\text {off }} \approx 5 \mathrm{mV}$; Curve 3: $1 \mu \mathrm{m}$-long high resistance strip, $R=140 \mathrm{k} \Omega\left(R_{\square} \approx 14 \mathrm{k} \Omega\right), V_{\text {off }} \approx 8 \mathrm{mV}$.

The low-resistive sample 1 showed insignificant nonlinearity of its $I-V$ curve, while the samples 2 and 3 (with $R_{\square} \approx R_{Q}$ or higher) exhibited a clear blockade region of current in their $I-V$ curves. The offset voltage $V_{\text {off }} \approx 5-8 \mathrm{mV}$ was approached at $V \approx 10-15 \mathrm{mV}$. We associate such characteristics of the samples with intrinsic Coulomb blockade effects (partially depressed by quantum fluctuations) in small Cr granules forming the strips. When a tunnel resistance of thin barriers separating the metallic granules increases up to the threshold value about $R_{Q}$, the array of these granules gradually turns to the blockade state. At lower resistances of the barriers, the quantum fluctuations completely wash out the charging effect. Assuming a uniform two-dimensional arrangement of the granules we can directly relate the sheet resistance $R_{\square}$ to the intergranular barrier resistance.

We made an attempt to use the gates to control the transport current through the strips, but the $I-V$ curves did not show noticeable dependence on the gate voltage in the significant range $\left|V_{g}\right| \leq 1 \mathrm{~V}$. This property can also be understood on the basis of the twodimensional array model: since the background charges $-e / 2 \leq Q_{0 i} \leq e / 2, i=1,2, \ldots, N$ are randomly distributed over the large number $N$ of granular islands, their additional polarization $\left(\propto V_{g}\right)$ produced by the common gate does not change the random character of their distribution. Therefore the $I-V$ curve remained almost the same when value of $V_{g}$ changed. (Note that for the same reason, the effect of $V_{g}$ on the $I-V$ curve of a one-dimensional array of 13 tunnel junction [8], [9] was not periodic and not as strong as in the case of transistor samples.)

\section{CHARACTERIZATION OF THE TRANSISTOR}

Our transistor structure comprised two sections of Crfilm strips with a sheet resistance $R_{\square} \approx 4 \mathrm{k} \Omega$. The total asymptotic resistance $R$ of the sample was about $110 \mathrm{k} \Omega$. This value is in fair agreement with the resistance of two Cr sections connected in series, $m R_{\square} \approx 104 \mathrm{k} \Omega$, where $m \approx 26$ is the total number of squares. This meant that the resistances of possible tunnel junctions at the $\mathrm{Al}-\mathrm{Cr}$ interfaces were seemingly small $\left(<R_{Q}\right)$. The resistance $R$ as well as the shape of the transistor $I-V$ curves were practically insensitive to the perpendicular magnetic field up to the strength $B=1 \mathrm{~T}$. (For that reason, most of the measurements were performed in a zero magnetic field.) At low temperature, $T \approx 30 \mathrm{mK}$, this value of $B$ ensured the normal state of $\mathrm{Al}$ and hence the normal density of states in the electrodes. The fact that the $I-V$ curves remained the same as those for superconducting $\mathrm{Al}$ electrodes substantiates our conclusion regarding the negligible role of the $\mathrm{Al}-\mathrm{Cr}$ interface resistance.

The current-voltage and gate-modulation curves of our transistor are presented in Fig. 3. These characteristics clearly demonstrate the typical single-electron behavior of the device: strong blockade effect (maximum size of blockade region $V_{\max } \approx 0.5 \mathrm{mV}$ ) and large modulation of the blockade by the gate. The zero bias resistance of the sample in the "open" state is measurable, about $60 \mathrm{M} \Omega$, and drastically decreases with the increase in $V$. The modulation curves are strictly periodic with a period $\Delta V_{g} \approx 7 \mathrm{mV}$. The constant amplitude and stable period persist over the whole range of measurements, viz. 40 periods.

From the characteristics obtained we conclude that the gate polarizes the island via the capacitance $C_{g}=$ 


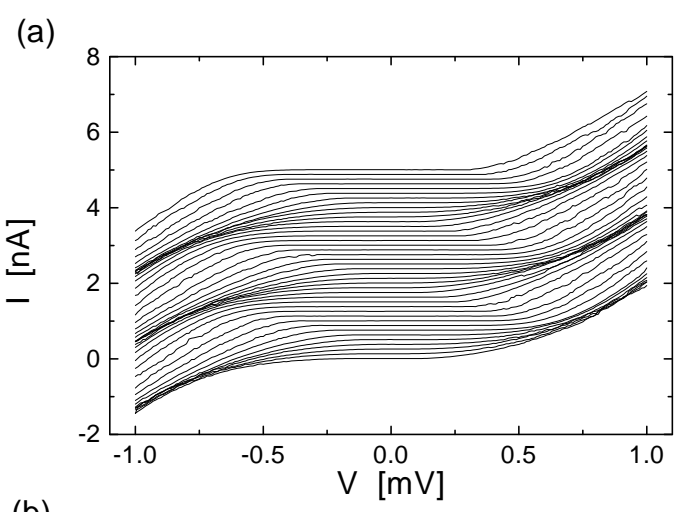

(b)

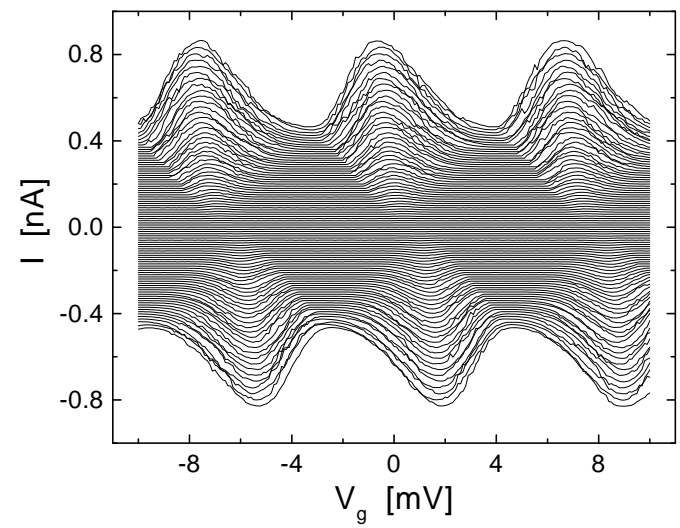

FIG. 3: Experimental $I\left(V, V_{g}\right)$ surfaces of the Cr-film transistor at $30 \mathrm{mK}$ : (a) $I-V$ curves measured at $V_{g}=0 \div 20 \mathrm{mV}$ with a step of $0.5 \mathrm{mV}$; (b) $I-V_{g}$ curves measured at $V=$ $-0.45 \div 0.45 \mathrm{mV}$ with a step of $0.01 \mathrm{mV}$.

$e / \Delta V_{g} \approx 23 \mathrm{aF}$. This value agrees with the sizes and mutual arrangement of island and gate. As long as the gate had a minor effect on the blockade in the detached strips, there is good reason to believe that the transistor gate does not change the conductance and the offset owing to $\mathrm{Cr}$ lines. Thus, the device operates in a similar way as the usual SET with tunnel barriers: the transport is realized by means of single electrons which arrive at (depart from) the island through highly resistive strips. The offset voltage of the transistor $V_{\text {off }} \approx 1.1 \mathrm{mV}$ was evaluated from the asymptotic characteristics of the $I-V$ curve taken at large $V>3 \mathrm{mV}$.

Having evaluated voltage-to-charge ratio $\mathrm{d} V / \mathrm{d} Q$ from the periodic dependence $V$ versus $V_{g}$, we measured noise spectra at different working points (on the slope, in the maximum and minimum) of the modulation curve at $I=5 \mathrm{pA}$. The data (the strongest noise on the slope) clearly pointed to the charge nature of the dominant noise. The shape of the spectrum was close to $1 / f$ and the charge noise level at $10 \mathrm{~Hz}$ was evaluated as $5 \times 10^{-4} e / \sqrt{\mathrm{Hz}}$. The value obtained is also typical of $\mathrm{Al} / \mathrm{AlO}_{x} / \mathrm{Al}$ transistors with the island size to be about $1 \mu \mathrm{m}$. We suppose that the appreciable noise in our

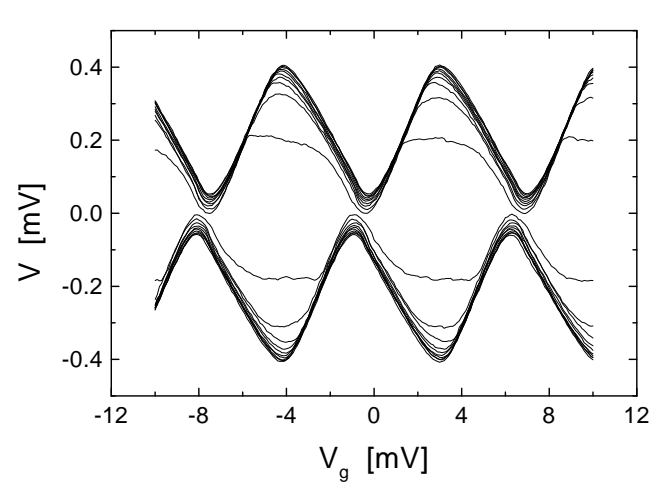

FIG. 4: $V-V_{g}$ modulation curves of Cr-film transistor measured for bias currents $I=0.1,0.2, \ldots 1 \mathrm{pA}$ at $T=30 \mathrm{mK}$.

structure is determined by the considerable contact area between island and substrate, and the figure can be improved by decreasing the sizes of the island [10].

\section{DISCUSSION OF RESULTS}

We found several remarkable features of the transistor sample investigated. In contrast to the property of traditional SETs, we observed significant nonlinearity of the $I-V$ curves in the open state, namely, $I \propto V^{\alpha}$, where $\alpha \approx 2.5$ (cf. the linear behavior of the tunnel barrier transistor). In the Coulomb blockade state the $I-V$ curve exhibits the large region with vanishingly small current (see, e.g., Fig. 3b). The current decay in the region of the blockade corner is substantially steeper $(\alpha>10)$ than the dependence $I \propto V^{3}$ which is typical of tunnel barrier transistors at low $T$ [11] because of the cotunneling effect [12]. This dependence indicates that the cotunelling process, i.e. arrival of an electron at the island and simultaneous escape of another electron from the island, is strongly suppressed. Such a suppression of cotunneling occurs in one-dimensional arrays of tunnel junctions with a large number of junctions [1] or in SETs supplied with miniature on-chip resistors, the so-called R-SET [13], due to the effect of a dissipative electromagnetic environment [14] created by these resistors. The latter system has the advantage to efficiently control the transport current by gate. It is interesting that - although our device is based on another junction type - its behavior is similar to that of R-SET, i.e. it combines the property of deep modulation of single electron current and considerable suppression of the cotunneling current.

Owing to the significant suppression of cotunneling, we were able to observe the $V-V_{g}$ modulation characteristics feeding a very small current $I$ through the transistor (see Fig. 4). For example, at $I=I_{0}=200 \mathrm{fA}$, the 
maximum value of $V$ approaches the value $\approx 0.3 \mathrm{mV}$. The evaluation of the cotunneling current in the symmetric tunnel-barrier SET device having similar parameters $\left(R=110 \mathrm{k} \Omega\right.$ and $\left.V_{\text {off }} \approx 1.1 \mathrm{mV}\right)$ at the same $V=0.3 \mathrm{mV}$ yields the value $I_{\text {cot }} \approx 15 \mathrm{pA}$, i.e. about two (!) orders of magnitude larger than $I_{0}$.

\section{SUMMARY.}

We demonstrated a new type of single-electron transistor with highly resistive Cr film strips instead of the traditional tunnel junction. Although a complete theory of its operation has not yet been elaborated, the device apparently behaves like a single electron system. It shows remarkable characteristics which are qualitatively similar to those of SETs with usual tunnel junctions. It is one of the advantages of our SET device that it is relatively easy to fabricate. The main difficulty consists in evaporating $\mathrm{Cr}$ film with a well-defined sheet resistance. The investigated device consists of elements rather large in size as compared to traditional tunnel junction SET devices. Therefore, there apparently is room for increasing the operating temperature of the metallic transistors without tunnel barriers by decreasing the sizes of strips and island.

Strong suppression of the cotunneling current, reasonable resistance in the open state and its insensitivity to the magnetic field are promising indications that this device can do the job of a low-leakage switch in delicate experiments with mesoscopic structures like single electron traps, pumps, etc. Another potential application of this transistor could be in digital SET circuits especially if the problem of the background charge is not as severe as in traditional SET devices. A further study of the background charge noise and drift in our devices is therefore to the foreground.

Finally, the metallic Cr-film transistors have a better tolerance as regards electric shock destruction because they have no tunnel barriers which are usually very sensitive to voltage pulses when tested.

\section{ACKNOWLEDGMENTS}

We wish to thank M.Yu. Kupriyanov for valuable discussions. This work was supported by the German
BMBF, the Russian Fund for Basic Research, the Russian Program "Physics of Solid State Nanostructures" and the Russian Ministry of Science.

[1] P. Lafarge, H. Pothier, E. R. Williams, D. Estive, C. Urbina, and M. H. Devoret, Z. Phys. B 85, 327 (1997).

[2] M. W. Keller, J. M. Martinis, A. H. Steinbach, and N. M. Zimmerman, IEEE Trans. on Instrum. and Meas., 46, 307 (1997).

[3] P. D. Dresselhaus, J. Li, S. Han, L. Ji, J. E. Lukens, and K. K. Likharev, Phys. Rev. Lett. 72, 3226 (1994).

[4] V. A. Krupenin, S. V. Lotkhov, D. E. Presnov, A. B. Zorin, F.-J. Ahlers, J. Niemeyer, H. Scherer, T. Weimann, and H. Wolf, Czech. J. Phys. 46, Suppl. 4, 2283 (1996).

[5] Y. Y Wei, J. Weis, K. V. Klitzing, and K. Eberl, Appl. Phys. Lett. 71, 2514 (1997).

[6] The idea that the single electron effects can occur in a metallic island connected to the electron reservoir by means of an "arbitrary scatterer" (not necessarily by a tunnel junction) was first proposed in: Yu. V. Nazarov, Phys. Rev. Lett. 82, 1245 (1999).

[7] J. Niemeyer, PTB Mitt. 84, 251 (1974); G. D. Dolan, Appl. Phys. Lett. 31, 337 (1977).

[8] L. S. Kuzmin, P. Delsing, T. Claeson, and K. K. Likharev, Phys. Rev. Lett. 62, 2539 (1989).

[9] P. Delsing, One-dimensional arrays of small tunnel junctions. In: H. Grabert and M. H. Devoret, editors, Single Charge Tunneling, pp. 249-274, New York, Plenum, 1992.

[10] V. A. Krupenin, D. E. Presnov, A. B. Zorin, and J. Niemeyer, J. Low Temp. Phys. 118, Nos. 5/6, 287 (2000).

[11] L. J. Geerligs, D. V. Averin, and J. E. Mooij, Phys. Rev. Lett. 65, 3037 (1990).

[12] The cotunneling process in SET transistors is associated with coherent tunneling of two electrons across two junctions simultaneously, see D. V. Averin and A. A. Odintsov, Phys. Lett. A 140, 251 (1989).

[13] A. B. Zorin, S. V. Lotkhov, H. Zangerle, and J. Niemeyer, J. Appl. Phys. 88, 2665 (2000).

[14] A. A. Odintsov, V. Bubanja, and G. Schön, Phys. Rev. B 46, 6875 (1992). 